\title{
Human induced vibration of staircases: Measurements and analysis
}

\author{
I. Kraincanic \\ London South Bank University, London, United Kingdom
}

A. Sparkes

Scott White and Hookins, Winchester, United Kingdom

\begin{abstract}
While there are numerous design guidelines for oscillations of floors, staircases have received comparatively less attention. Dynamic testing of existing staircases can improve understanding of their behaviour and lead to more accurate methods for predicting accelerations in service. This study uses a smartphone device to measure oscillations of a helical staircase. The smartphone accelerometer's output was compared to the output of a laboratory accelerometer and in the range of frequencies relevant to this study the differences were negligible. A helical staircase was tested in heel drop and walking tests to find natural frequencies and damping of a staircase. Computer analysis results for natural frequencies were in good correlation with the measured data. The results of footfall analysis showed that despite a relatively high fundamental frequency of the staircase, its response factor exceeded the one prescribed by the design guidelines. Footfall analysis results depend critically on the correct estimate of the damping ratio. The actual damping ratio was found to be higher than the one in guidance documents.
\end{abstract}

\section{INTRODUCTION}

\subsection{Vibration of stairs}

Steel staircases are flexible structures with a low mass subjected to high dynamic loads and hence likely to have high vibration levels. In addition, fully welded steel staircases can have the damping ratio as low as $0.5 \%$ (Smith (2009)). Any staircase with a natural frequency less than $10 \mathrm{~Hz}$ is likely to experience unacceptable levels of vibration (Kerr and Bishop (2001)).

The response to dynamic excitation is typically measured in terms of acceleration. The root-meansquare acceleration $a_{\text {rms }}$ effectively assesses how long a system experiences a level of vibration. This value is divided by a baseline RMS value set out in both the ISO 10137:2007 and BS 6472-1:2008 for a given frequency, which corresponds to the threshold value of human perception of vibration. The resulting response factor can then be compared against the limiting multiplying factors of 24 for heavy use and 32 for light use staircases (Bishop et al. (1995)).

Staircases experience much higher loads than the floors in general and a fast stair decent can normally be assumed with a footfall rate of between 3 and 4 per second and even up to 4.5 footfalls per second (Bishop et al. (1995)). Kerr and Bishop (2001) showed that only the first two harmonics of walking activity induced on stairs need to be considered, and proposed the corresponding Fourier coefficients.

Based on research findings, SCI P354 (Smith et al. (2009)) and Concrete Centre document (Willford and Young (2006)) provide guidelines for design of stairs to footfall oscillations. The latter gives practical advice on dynamic testing of staircases, including heel drop and walking tests.

\subsection{Dynamic testing of staircases}

In heel drop and walk-by tests of steel and reinforced concrete stairs, the natural frequencies of about 50 and $75 \mathrm{~Hz}$ were found, respectively (Kim et al. (2008)). All tested staircases had response factor limits within acceptable levels. An impact hammer was used in a study by Davis and Avci (2015) to apply dynamic load in order to more accurately estimate modal properties of a slender monumental staircase.

Modern smartphones possess sensitive enough accelerometers and along with specifically developed applications could be used to carry out insitu dynamic testing (Lacy et al. (2015)). The limitations are the phone accelerometers' limited resolution and sampling rate, however smartphones may be ideal for lightweight or flexible structures subject to footfall vibrations. A comparison between the sensitivity of various mobile device accelerometers and professional accelerometers 
showed that mobile devices with a higher resolution accelerometer were less disturbed by noise and thus resulted in a more accurate recording of the vibration signal (Feldbusch, et al. (2017)).

Smartphone devices have been used for structural health monitoring (SHM) to measure the dynamic response, because they are inexpensive and can be connected wirelessly (Fang, et al. (2016)). The authors experimentally verified the use of smartphones for the purpose of SHM using a threestory shear frame attached to a shake table with smart phones attached to each level with corresponding accelerometers. The table was then excited through a range of frequencies and the results compared against each other, and it was concluded that smartphones accurately recorded the dynamic response compared to the accelerometers.

In this work, dynamic testing is carried out on the helical staircase using heel drop and walk-by tests as described in Wilford and Young (2006). A smartphone with VibSensor application is used to record and analyse the acceleration data. The staircase structure is modelled in Autodesk Robot Structural Analysis (RSA) program, capable of calculating modal properties of the staircase and also the response factor using the footfall analysis method described in SCI P354.

\section{DYNAMIC TESTING}

\subsection{Description of the helical staircase}

The helical steel staircase is located in a cylindrical concrete core from which it is supported by steel cantilever plate beams that are bolted into the concrete walls (Figure 1). These cantilever supports are located at each end of the landings with one located mid-flight of each staircase, six per full flight of stairs. The stair treads, risers, stringers and landings are constructed out of $15 \mathrm{~mm}$ steel plate to form a fully welded steel staircase. The staircase treads and landing areas have a thin vinyl covering and aluminium nosing protection. Sizes and dimensions were taken from technical drawings and measurements taken directly on the staircase.

\subsection{Calibration}

The readings from the smartphone's accelerometer were compared with the readings from a professional accelerometer for a range of frequencies from 1 to $50 \mathrm{~Hz}$. The phone and an accelerometer were placed onto a mechanical vibrator platform and fixed down securely with tape. The mechanical vibrator was connected to a function generator. The generator was tuned to a specific frequency and the readings were taken by both the smartphone and the accelerometer.

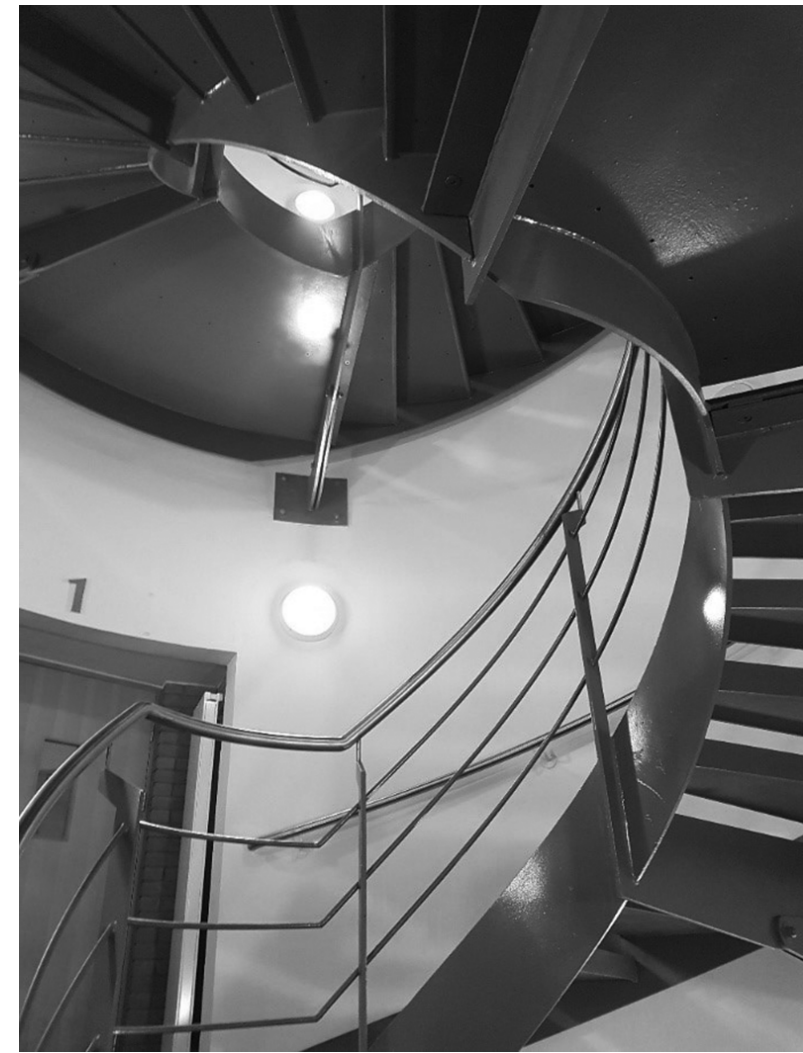

Figure 1. Helical staircase with cantilever supports

The results showed a small (less than 5\%) discrepancy for frequencies between 3 and $9 \mathrm{~Hz}$, with practically zero difference for $10 \mathrm{~Hz}$ and above. In the range $0-2 \mathrm{~Hz}$, however, the difference was significant.

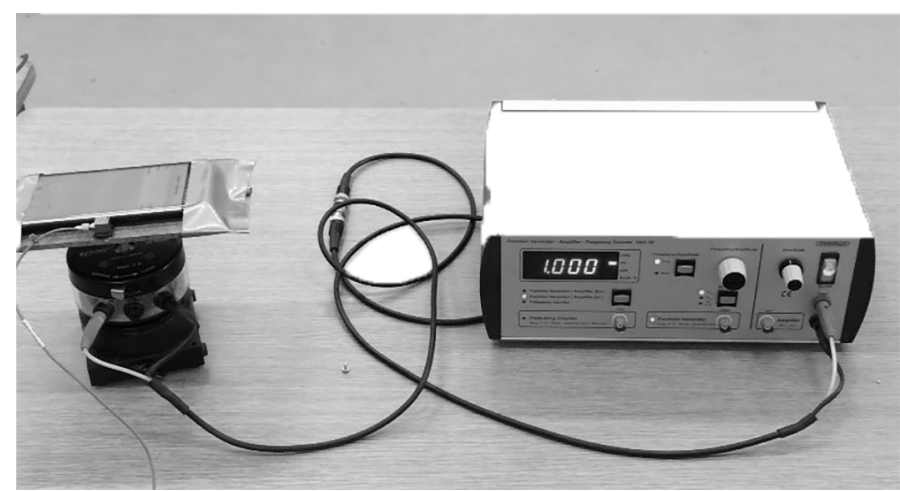

Figure 2. Calibration of the smartphone's accelerometer

\subsection{Heel drop tests}

For a heel drop test, acceleration data were recorded as a time history graph. This time history was then converted into the frequency domain using Fast Fourier Transform (FFT), to produce acceleration versus frequency spectra from which the modal (natural) frequencies of the structure can be identified.

Once the phone was in position and attached to the stair, the VibSensor application was opened. A ten second recording period was set with a 5 second 
delay to minimise any interference from pressing the start icon. A heel drop was then performed by a person $(80 \mathrm{~kg})$ raising up on their toes and then dropping down to their heels. For each test, the phone was positioned near the inner edge of the stair case, midway between cantilever supports where the biggest vibration response was likely to occur.

The acceleration graph from the middle landing is in shown in Figure 3. From the data it is possible to estimate the fundamental frequency which was 27 Hz. The damping ratio could be estimated approximately, from the logarithmic decrement of damping, as $8 \%$. In the frequency domain result for the middle landing in Figure 4, the fundamental frequency of $27 \mathrm{~Hz}$ is prominent.

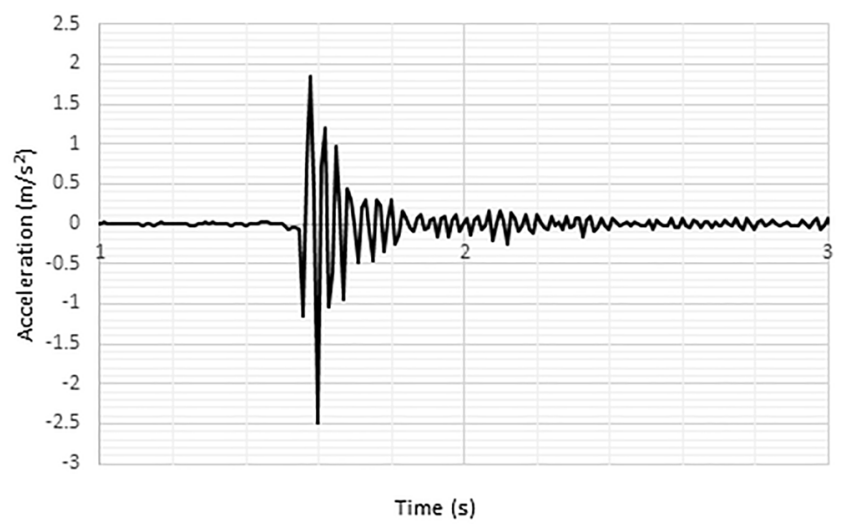

Figure 3. Heel drop time history for the middle landing

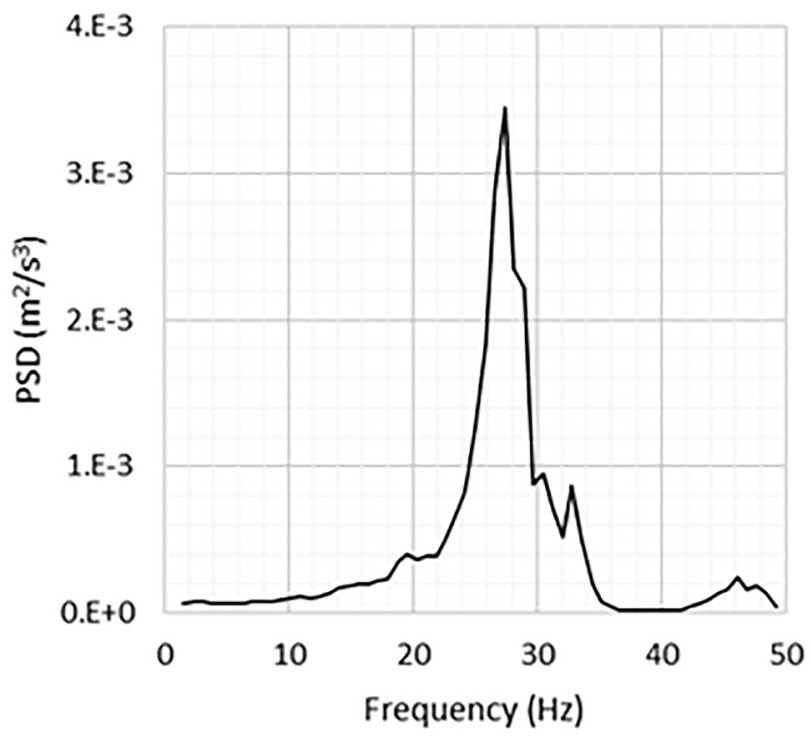

Figure 4. Heel drop frequency spectrum for the middle landing

Frequency spectrum for the bottom landing and the stair near the bottom landing in heel drop tests are shown in Figures 5 and 6 respectively.

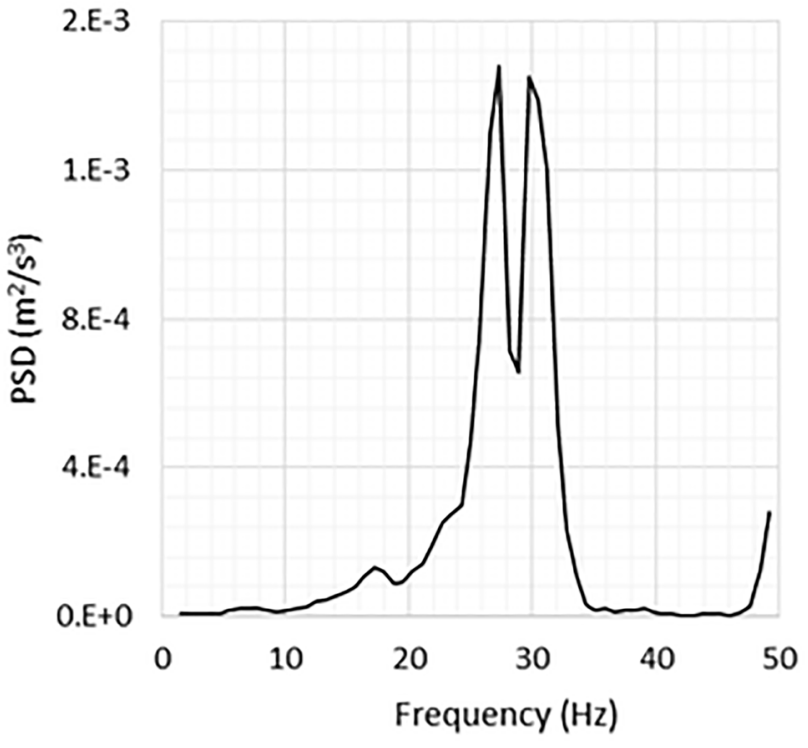

Figure 5. Heel drop frequency spectrum for the bottom landing

It is evident from comparing Figures 4 and 5 that the bottom (and top) landing are oscillating in both the first and second mode, while the middle landing oscillates significantly in the first, but not the second mode. They both show $27 \mathrm{~Hz}$ as the fundamental frequency, and the bottom landing has $32 \mathrm{~Hz}$ as the next peak value.

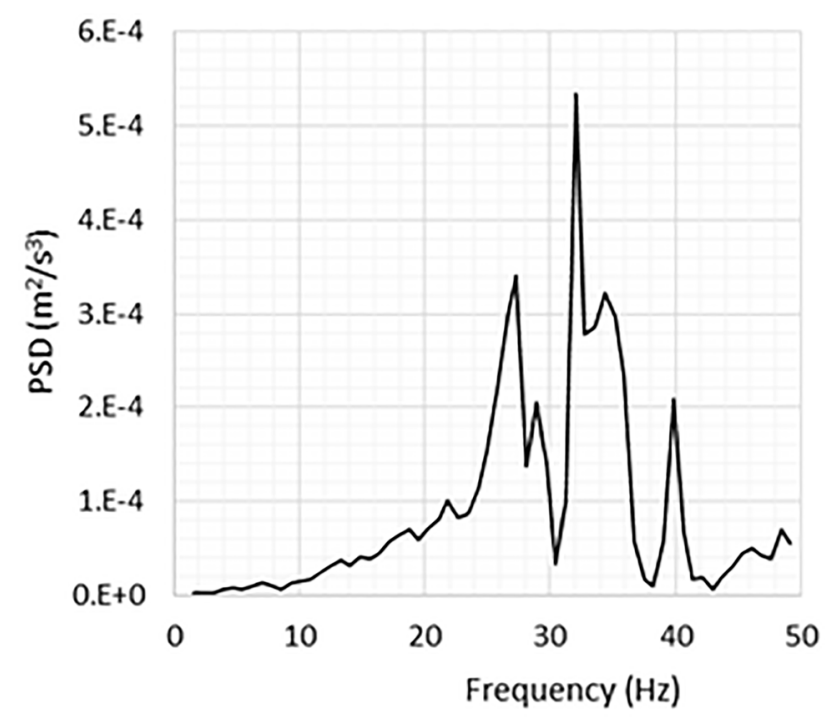

Figure 6. Heel drop frequency spectrum for the stair near the bottom landing

For the stair near the bottom landing heel drop test, shown in Figure 6, the second mode value is dominant, while for the stair near the middle landing, the first frequency has a higher PSD value. The mode of vibration of individual stairs can be seen here, with a frequency of $40 \mathrm{~Hz}$. 


\subsection{Walk-by tests}

The walk-by tests consisted of a person $(80 \mathrm{~kg})$ walking up and down one full story flight of the staircase between the third and fourth floor's landing at two different pacing frequencies of $1.8 \mathrm{~Hz}$ ascending and $2.7 \mathrm{~Hz}$ descending. These pacing frequencies were chosen as integer multiples of the natural frequency found from the heel drop tests $(27$ $\mathrm{Hz}$ ) likely to build up a resonant response. They lie within the range of typical pacing frequencies stated in SCI P354 for staircases of $1.2 \mathrm{~Hz}$ to $4.5 \mathrm{~Hz}$. The pacing frequency for each test was controlled with the use of the Google metronome feature on another smartphone.

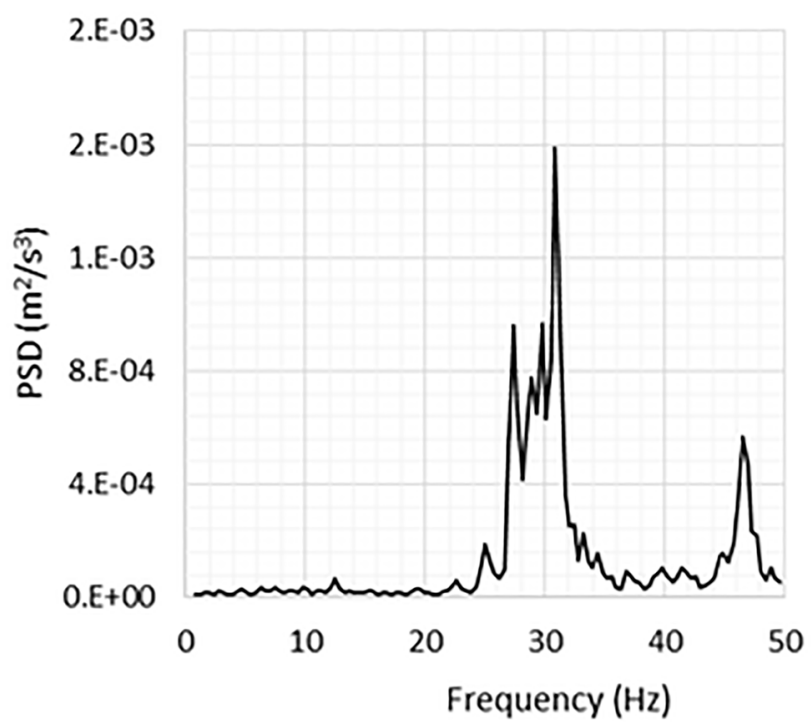

Figure 7. Walk by frequency spectrum for walking downstairs at $2.7 \mathrm{~Hz}$

Walking tests up and downstairs showed similar frequency spectrum, with the fundamental frequency of $27 \mathrm{~Hz}$. In the acceleration graphs there was no evidence of a resonant response, with individual steps shown as impulses which died down.

\section{COMPUTER ANALYSIS}

\subsection{Computer model}

Computer model was created based on the engineering general arrangement drawings and measurements taken from the staircase in RSA program. Modal and footfall analysis were carried out using the inbuilt facilities of the software. The landing was modelled as an isotropic $15 \mathrm{~mm}$ thick shell element. In one version of the model, stairs were represented as continuous orthotropic shell elements with unidirectional ribs. In another, which was adopted, stairs were modelled as individual beams of the tapered width. The footfall analysis parameters were set by selecting 'Stairs SCI P354 (2007)' for the Excitation forces. Damping ratio was set to $8 \%$, which was the value estimated from the heel drop test.

\subsection{Results from modal analysis}

Table 1 shows the natural frequencies from RSA modal analysis. An imposed point load of $0.80 \mathrm{kN}$ was applied at the same locations as the heel drops. The fundamental natural frequency of $25.33 \mathrm{~Hz}$ differs from the $27 \mathrm{~Hz}$ obtained from the heel drop test by $6 \%$. A possible reason for this underestimation of the natural frequency could be related to the accuracy of the finite element model. Modelling of the stair treads and risers as beams could be reducing the stiffness of the model. There was an additional mode shape for cases with the point load added. Mode 4 in all cases corresponded to the vibration of individual stairs. In mode 1, bottom, top and middle landing moved in the same direction, while in the mode 2 (shown in Figure 8), top and bottom landing move in opposite directions, while the middle one does not move noticeably.

Table 1: Natural frequencies from RSA output

\begin{tabular}{llll}
\hline Mode & No point load & $\begin{array}{l}\text { Point load on } \\
\text { bottom landing } \\
(\mathrm{Hz})\end{array}$ & $\begin{array}{l}\text { Point load on } \\
\text { middle landing } \\
(\mathrm{Hz})\end{array}$ \\
\hline 1 & 25.33 & 24.78 & 24.83 \\
2 & - & 27.79 & 28.08 \\
3 & 29.41 & 30.00 & 29.49 \\
4 & 43.51 & 43.51 & 43.51 \\
\hline
\end{tabular}

\subsection{Footfall analysis}

The resonant and transient response factors calculated from the footfall analysis in RSA are shown in Figures 9 and 10. This highlighted visually where the areas with highest response factors were, centrally on the top, bottom and middle landing, and on the inner side of stairs, furthest from the supports. The maps show the resonant and transient response factors, the maximum being 17.3 and 96.7 respectively. While the resonant response factor is within acceptable limits, the transient one is considerably higher than the limiting value given in the codes (32 for light use staircase). The prediction of response factors depends critically on the correct estimation of the damping ratio for the structure. In this case, it was found to be $8 \%$, while the guidance for welded steel staircases is $0.5 \%$. Using the latter value would have resulted in overestimation of response factors. 


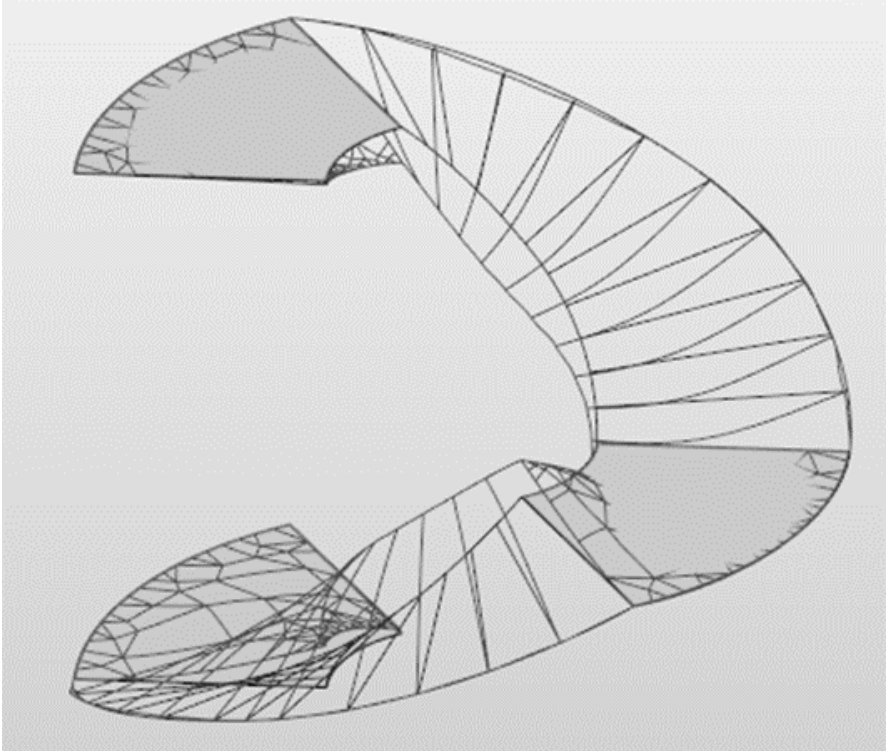

Figure 8. Mode 2 of vibration at $29.4 \mathrm{~Hz}$, from RSA modal analysis output

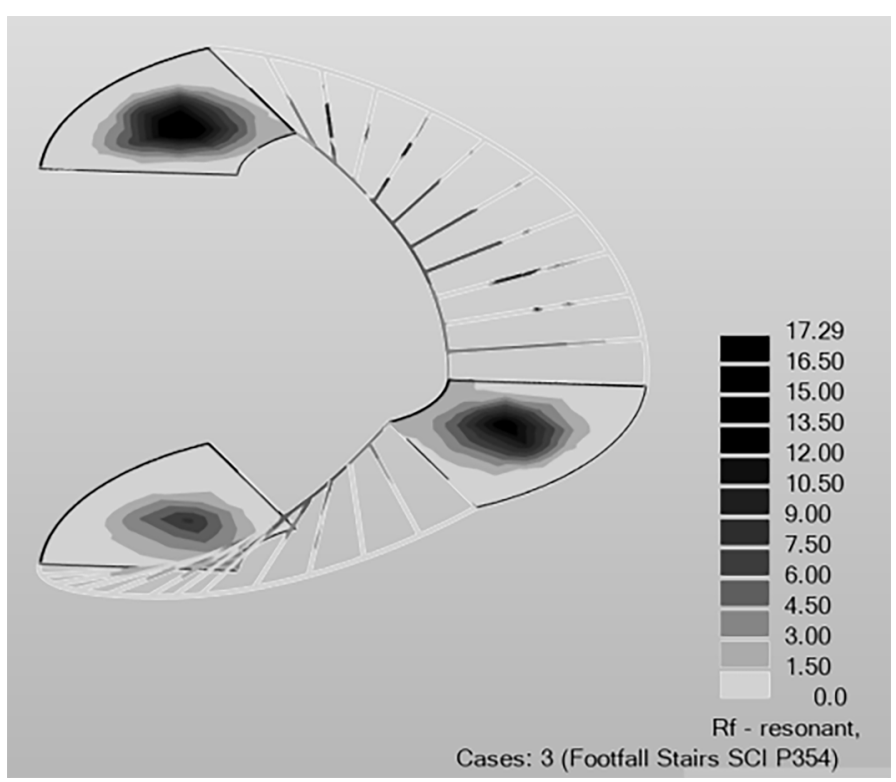

Figure 9. Resonant response factor from RSA footfall analysis

\section{CONCLUSIONS}

This study shows that measurements from a smartphone accelerometer can be used to assess dynamic response of a structure within the range of 3 to $50 \mathrm{~Hz}$. Results showed that even a staircase with a relatively high fundamental frequency may experience significant levels of acceleration from human excitation. Correct estimate of damping is crucial for the prediction of floor acceleration in service. The VibSensor app provides the timeacceleration as well as frequency spectrum graphs, thus enabling quick processing of data. Testing existing structures to dynamic impulse and harmonic load can be used to verify computer models and improve their accuracy, in order to be able to predict dynamic behaviour of a structure in design stage. Although this research focused on a quite specific structure, the methods presented here could be adopted for different types of structures susceptible to human induced oscillations.

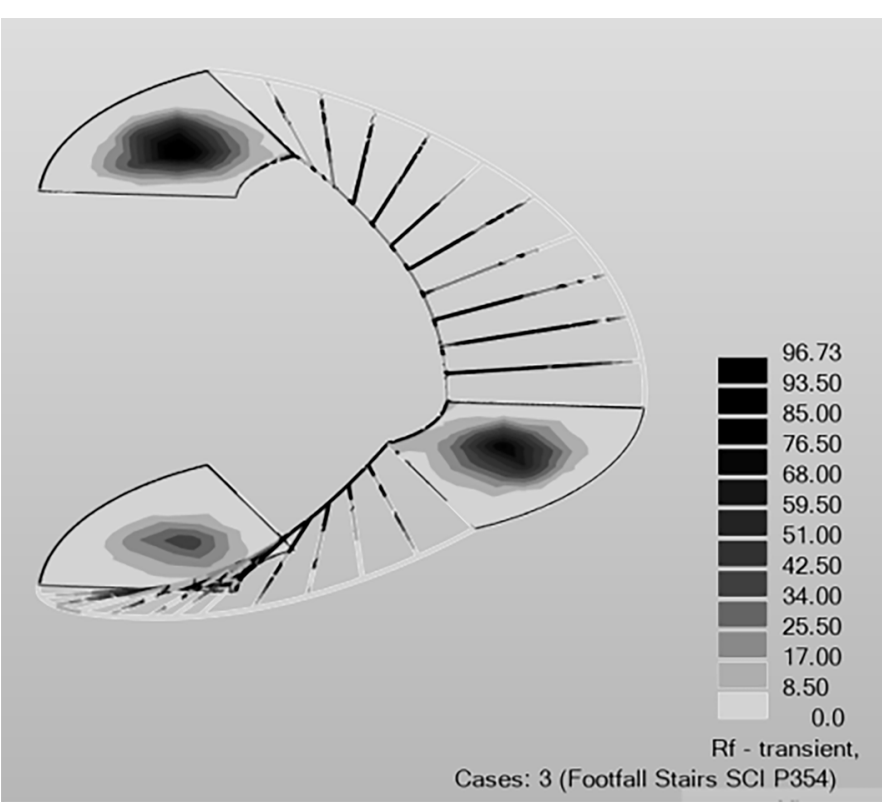

Figure 10. Transient response factor from RSA footfall analysis

\section{REFERENCES}

Bishop N.W.M, Willford M. \& Pumphrey R. 1995. Human induced loading of flexible staircases. Safety Science: 261276.

BS 6472-1:2008 Guide to evaluation of human exposure to vibration in buildings - Part 1: Vibration sources other than blasting. London: British Standards Institute.

Davis, B. \& Avci, O. 2015. Simplified vibration serviceability evaluation of slender monumental stairs. Journal of Structural Engineering 141(11).

Fang, K., Tian, J.D., Zhang, D.Y. \& Li, H., 2016. Smartphones equipped with android application software for structural health monitoring. Proceedings of the International Conference on Smart Infrastructure and Construction: 271275.

Feldbusch, A., Sadegh-Azar, H. \& Agne, P., 2017. Vibration analysis using mobile phone devices (smartphones or tablets). Procedia Engineering 199: 2790-2795.

ISO 10137:2007 Bases for design of structures - Serviceability of buildings and walkways against vibration. 2 ed. Geneva: International Standards Organisation.

Kerr, S.C. \& Bishop, N.W.M. 2001. Human induced loading on flexible staircases. Engineering Structures 23(1): 37-45.

Kim, S.B., Young H.L., Scanlon, A., Kim H. \& Hong K., 2008. Experimental assessment of vibration serviceability of stair systems. Journal of Constructional Steel Research 64: 253259.

Lacy, A.D., Parker, J. \& Winslow, P., 2015. Do-it-yourself dynamics: testing of the staircase at Institution HQ. The Structural Engineer (July): 48-51. 
Smith, A. 2009. AD 330 Vibration of steel staircases. Acot: Steel Construction Institute.

Smith, A.L., Hicks, S.J. \& Devine, P.J., 2009. SCI P354 Design of Floors for Vibration: A New Approach. Revised ed. Ascot: The Steel Construction Institute.

VibSensor smartphone application user guide. 2017. Now Instruments + Software.

Willford, M.R. \& Young, P., 2006. A Design Guide for Footfall Induced Vibration of Structures. Surrey: The Concrete Society. 